\title{
Informational Entropy as a Source of Life's Origin
}

\author{
Shahram Yazdani \\ Department of Pediatrics, David Geffen School of Medicine at University of California, Los Angeles, CA, USA \\ Email: Syazdani@medn et.ucla.edu
}

How to cite this paper: Yazdani, S. (2019) Informational Entropy as a Source of Life's Origin. Journal of Modern Physics, 10, 1498-1504.

https://doi.org/10.4236/jmp.2019.1013099

Received: October 16, 2019

Accepted: November 10, 2019

Published: November 13, 2019

Copyright (c) 2019 by author(s) and Scientific Research Publishing Inc. This work is licensed under the Creative Commons Attribution International License (CC BY 4.0).

http://creativecommons.org/licenses/by/4.0/ (c) (i) Open Access

\begin{abstract}
While the second law of thermodynamics suggests that our universe is driven by the tendency towards disorder, living organisms seem to exempt themselves by creating physiologic complexity. Since genetic material is life's blueprint, better understanding of the origins of life is predicated on deciphering the conditions that allowed the formation of this complex molecule with its unique properties. In this article, we propose and examine the hypothesis that informational entropy models would allow for the formation of complex organic molecules with genetic properties, without the disruption of the second law of thermodynamics. Therefore, we demonstrate that formation of life's blueprint may have initially been derived by informational entropy by means of decomplexification of the materials with higher informational entropy content, leading to the formation of primitive genetic molecules.
\end{abstract}

\section{Keywords}

Entropy, Informational Entropy, Genetic Material, Second Law of Thermodynamics

\section{Introduction}

The ability of living organisms to defy the natural tendency of all matter towards decay by creating order and evolving into complex organisms has been the subject of much fascination since the ancient times. Perhaps the earliest implementation of the role of disorder in relation to life was conveyed to us through Greek mythology where Gaea, the earth and mother of all things, sprang from Chaos. During the more modern times, the eighteenth century German chemist and physician Georg Ernst Stahl described life as a conservatio mixtionis corporis, against the tendency to decompose. Later, Erwin Schrodinger in his 1944 book titled "What is life", described the tendency of the living organisms to seemingly 
exempt themselves from the second law of thermodynamics by avoiding decay through a state of non-equilibrium with their surroundings [1]. Thus, while our universe is driven by the tendency towards higher entropy or disorder, living organisms constantly strive to increase or maintain physiological complexity in order to avoid the increase in their entropy to a state of equilibrium with the surrounding environment. As thermodynamically open systems, living organisms must harness free energy or enthalpy from food and chemical substrate to create an ideally equal balance between the entropy created within the system and the entropy exported [2]. Thus, as Schrodinger had concluded, all living organisms continuously create and export positive entropy while maintaining as he called it, "negative entropy". Furthermore, since the transformation of inorganic compounds to organic compounds was initially demonstrated in 1828 by the German chemist Friedrich Wöhler, who artificially obtained urea by treating silver cyanate with ammonium chloride, the transformation of inanimate being to life form is no longer hindered by the absence of preceding organic compounds. Thus, a better understanding of the forces that may resolve the classical thermodynamic dilemmas of life forms, paves the way for peering into possible conditions and requirements that may have initially led to the formation of life, or at the very least, its most fundamental component, life's genetic blueprint (e.g. DNA, RNA).

Yet, formation of life's molecular blueprint and its surrounding structures from the less complex substrates requires temporary violation of the second law of thermodynamics by reducing the entropy of the substrates through their organization into more complex compounds and eventually a living organism with the promise of increased consumption of food material, export of entropy, and thus overall increase in the system's entropy. To overcome this challenge, Horowitz and England have proposed an in silico model of molecules where inanimate material settings may act "life-like" by maintaining a "far-from-equilibrium" steady state [3].

In this article, we demonstrate that information has physical properties that provide an essential component of the thermodynamics of living organisms and can provide a solution in regards to the thermodynamic pathway that paved the way to de novo formation of life from inanimate organic or inorganic substrates. In other words, we demonstrate that the initial formation of the primitive genetic material, which is one of the most fundamental components of life, is not formed through a series of complex physical reactions that are only applicable in theoretical timeframes or environments, but in fact is a natural phenomenon that can be explained through the observed reactions within the physical environment.

Therefore, we propose that,

1) Shannon's informational entropy in the context of biology, suggests that information is an essential deriving factor in the formation of life.

2) Inanimate inorganic compounds can not only provide a substrate for life, but also provide informational entropy content for the earliest forms of genetic 
material, thus providing a solution for the thermodynamic derive that led to the formation of life.

\section{Information and Life}

Life's defiance of the second law of thermodynamics within its system has led to the exploration of alternative or complementary physical laws that may explain life's tendency to thrive through the export of entropy to its surrounding in order to maintain disequilibrium between the living organism, and its surrounding environment.

As Sanchez has demonstrated through the "egg conundrum", which calculates the thermodynamics of a fertilized egg compared to an unfertilized one, the statistical and classical thermodynamics cannot explain the formation of life [4]. Furthermore, Sanchez calculates the assignable statistical entropy content of each codon using Gibbs entropy formula.

$$
S=-k \sum_{l} P l \ln (P l)
$$

where $k$ is the Boltzmann's constant, $P l$ in this setting represents the probability of a unique consecutive "linear" sequence of $\mathrm{N}$ codons in the $I_{t h}$ state. Using this approach, Sanchez concludes that the negative entropy assignable to each codon is estimated as,

$$
S=-3.06 \mathrm{~K}
$$

As the equation above demonstrates, the statistical negative entropy content of each codon does not fully account for the positive entropy generated by an organism [4]. Therefore, inclusion of the informational content of DNA is essential to account for its total negative entropy content.

However, while including the informational content of DNA as a source of its negative entropy offers a solution for the inadequacy of the statistical thermodynamic models, it implies that formation of genetic material requires increase in stored information, which further poses the question as to what conditions originally derived formation and accumulation of information in the initial molecular blueprints that would eventually lead to life formation.

\section{Substrate for Information}

To better understand conditions under which the primitive form of a complex, information-rich molecule such as primitive RNA or DNA may have been initially formed, we propose a look at the informational content of some of the comparatively more complex inorganic compounds found in nature.

We begin by introducing the Shannon information formula

$$
H=-\sum_{i=1}^{s} P i \log _{2} P i \text { (bits/symbol) }
$$

where $H$ is the amount of information contained in a system with $n$ symbols of $s$ different types occurring with the probability $P i$. The importance of this formula is its universal application to any form of message, regardless of its medium. 
Therefore, blueprint of living organisms and inanimate sources share certain commonalities in the rules governing information and its relation to physical properties as described in the example below.

Consider the example of Crystals. As Krivovichev has demonstrated, the structural complexity and configuration of crystal structures add informational entropy to crystal structures that go beyond the molecular content of each crystal [5]. The informational content $I_{G}$ per atom appearing in a crystal is calculated as,

$$
I_{G}=\sum_{i=1}^{k} P i \log _{2} P i \text { (bits/atom) }
$$

where $k$ is the number of crystallographic orbits, denoted as $i$, and $P i$ is the probability of a randomly chosen atom appearing in the $i$-th crystallographic orbit.

Using Shannon's information equation, Krivovichev demonstrates that even in a simple crystal with all atoms equivalent and distributed into various asymmetrical crystallographic orbits, the configuration of orbits and arrangement of various crystal "cells" creates informational content that in fact holds physical properties, makes negative contribution to the entropy of the crystal, and therefore, should be accounted for in the total entropy of the crystal.

\section{Information as the Common Denominator}

As Equations (2) and (4) imply, DNA and crystals carry information through the arrangement of their analogous informational building blocks. More specifically, the configuration of atoms in a crystal carries information, similarly to the linear arrangement of codons in the case of a strand of DNA. Furthermore, as Landauer suggested (1961, 1996), erasure of each bit of information requires energy loss (expressed in degrees Kelvin) and thus, information and energy are interlinked [6] [7].

$k T \ln 2$

where $K$ is Boltzman's constant and temperature is in Kelvin.

As Landauer suggests, for each bit of information lost at Temperature $T, k n 2$ of energy is released into the environment, which in turn contributes to the entropy $S$ of the environment.

This suggests that complexification of a crystal, and formation of any form of life's blueprint should both be considered within the context of energy and information exchange, within their surrounding environment.

Considering the information-rich content of many complex crystals, it is conceivable that conditions that allowed information and thermodynamic exchange between the substrates of the primitive genetic material and comparatively more complex crystals in their surroundings, led to the formation of the earliest genetic material [7]. Therefore, the net result of the simplification of these crystal structures, and hence increase in their informational and thermodynamic entropy, led to complexification and decrease in the thermodynamic and informa- 
tional entropy of the substrates of the earliest forms of codons. Hence, this transference of negative informational and thermodynamic entropy from the comparatively more complex molecules such as complex crystals to genetic substrates, eliminates the need for a hypothetical temporary decrease in the entropy of the system with the promise of eventual increase in the entire system's entropy that would arrive after the complete formation of a functioning organism. Furthermore, considering the unknown composition of the initial blueprints, minerals of complex composition may have in fact provided both the substrate and entropy (informational and statistical) of these molecular blueprints. Therefore, we propose that inorganic molecules may have potentially, not only provided the substrate to form organic molecules, as Friedrich Wöhler had demonstrated, but also acted as the source of informational entropy through a series of chemical reactions that led to decomplexification of these inorganic molecules coupled with transference of their informational entropy to the newly formed organic molecules. Thus, this exchange of informational entropy can satisfy the second law of thermodynamics while providing adequate informational entropy to allow these primitive molecules to carry gene-like properties.

However, the proposed hypothesis greatly hinges upon the existence of inorganic molecules (e.g. mineral crystals) with complex structures that would have been geologically formed prior to the formation of the first life form, while containing adequate complexity to contain informational entropy that exceeds the primitive genetic material.

Although the informational content and composition of the earliest genetic material is beyond the scope of this paper and may have significantly varied from the informational content of the modern DNA, a comparison of the more primitive crystals' informational content with the more modern DNA codons provides some clues as to the plausibility of this hypothesis. Therefore, if a unit cell of a crystal holds more information than a comparable DNA codon, then it is possible that decomplexification of an analogous crystal could have led to the formation of a codon of comparatively lesser informational content and complexity, and therefore, the second law of thermodynamics is preserved.

To compare the informational content of a unit of crystal with the entropy of each codon of DNA, we must first consider the energy content of each bit of information as noted in Landauer's equation. The ratio of the energy released and therefore contributed to the entropy of its environment from all or part of the informational content of a crystal in the process of decomplexification as calculated through Landauer's equation, divided by the Gibbs entropy of each codon of DNA must be equal or exceed 1.

$$
\begin{gathered}
I_{c}(k T \ln 2) / 3.06 k=I_{c} T 0.227>1 \\
I_{c} T>1 / 0.227=4.405
\end{gathered}
$$

where $I_{c}$ is the informational content of a unit of crystal, $k$ is the Boltzman's constant and $T$ is the temperature of the environment in Kelvin. As this indicates, as long as the product of the temperature and informational content of each unit 
cell of a crystal exceeds 4.405 , the ratio above is satisfied.

A brief review of some of the structurally simple crystals in the initial geological formations $(>4.55 \mathrm{Ga})$ preceding our estimated time for the formation of primitive life indicates that a variety of crystals had moderate level of informational content that may have reached up to 200 bits/unit cell, far exceeding the informational content needed to form each codon of a strand of DNA [8] [9].

Minerals and their crystal structure also offer a unique solution since despite their low entropy and high informational content, they are inevitably formed and evolve into more complex structures under geological conditions [9]. Therefore, geological conditions that naturally led to the formation of complex inorganic structures such as minerals, may have in turn led to the formation of life and its essential components through a series of complexification (i.e. crystal formation), and subsequent decomplexification of crystals that lead to complexification of inorganic and organic molecular building blocks of the initial genetic material.

\section{Conclusion}

Statistical thermodynamic models alone fail to explain the conditions under which life could have initially been formed. However, integration of the informational entropy models provides a plausible scenario for life formation under known natural conditions. This model suggests that informational and energy exchange between the initial substrates of the primitive genetic material and the more complex molecules and structures may have made life possible. The presence of crystals and minerals with low entropy and high informational content preceding life formation, offers a plausible source of such complex molecules. Further experimentation is needed to test this hypothesis by delineating the potential chemical reactions through which inorganic molecules in the environment surrounding the newly formed organic molecules can exchange and contribute informational entropy, in order to enable gene-like properties that are essential for life formation.

\section{Author Contributions}

The sole author of this article has been actively involved in forming this hypothesis and writing the final copy of this manuscript. The author takes full responsibility in the integrity of this study and its content. The author has read the submitted manuscript and is fully aware and in agreement with the accuracy of the presented information and conclusions of this study.

\section{Competing Financial Interest Statement}

The author of this article has no financial conflicts of interest in relation to the outcomes of this study. This study has been financed through internal sources.

\section{Conflicts of Interest}

The author declares no conflicts of interest regarding the publication of this paper. 


\section{References}

[1] Schrödinger, E. (1944) What Is Life? Cambridge University Press, Cambridge.

[2] Prigogine, I. (1947) Etude thermodynamique des Phenomenes Irreversibles. Desoer Liege.

[3] Horowitz, J.M. and England, J.L. (2017) Proceedings of the National Academy of Sciences of the United States of America, 114, 7565-7570. https://doi.org/10.1073/pnas.1700617114

[4] Sanchez, I.C. (2011) Journal of Modern Physics, 7, 654-657. https://doi.org/10.1073/pnas.1700617114

[5] Krivovichev, S.V. (2016) Acta Crystallographica, 72, 274-276. https://doi.org/10.1107/S205252061501906X

[6] Landauer, R. (1961) IBM J. Res., 5, 183-191. https://doi.org/10.1147/rd.53.0183

[7] Landauer, R. (1996) Physics Letters A, 217, 188-193. https://doi.org/10.1016/0375-9601(96)00453-7

[8] Krivovichev, S.V. (2013) Mineralogical Magazine, 77, 275-326. https://doi.org/10.1180/minmag.2013.077.3.05

[9] Hazen, R.M., Papineau, D., Bleeker, W., Downs, R.T., Ferry, J.M., McCoy, T.J., Sverjensky, D.A. and Yang, H. (2008) American Mineralogist, 93, 1693-1720. https://doi.org/10.2138/am.2008.2955 